\title{
Concrete wave barriers to mitigate ground vibrations induced by railway traffic: a three-dimensional numerical study
}

\author{
Jesús Fernández-Ruiz (Main and Corresponding author) \\ Universidad de A Coruña \\ University of La Coruña, Department of Civil Engineering \\ Campus de Elviña, s/n, 15071, La Coruña (Spain) \\ jesus.fernandez.ruiz@udc.es \\ https://orcid.org/0000-0002-6201-4235

\section{Luis Medina Rodríguez} \\ Universidad de A Coruña \\ University of La Coruña, Department of Civil Engineering \\ Campus de Elviña, s/n, 15071, La Coruña (Spain) \\ luis.medina@udc.es \\ https://orcid.org/0000-0002-1864-4360 \\ Manuscript Code: 13978 \\ Date of Acceptance/Reception: 09.11.2020/07.01.2020 \\ DOI: 10.7764/RDLC.19.3.395
}

\begin{abstract}
Continuous wave barriers are mitigation measures to reduce vibrations induced by railway traffic which have been well studied in technical literature. Nevertheless, there are not many studies about discontinuous concrete wave barriers. By this reason, in this paper continuous and discontinuous concrete wave barriers are studied and compared. With this objective, two theoretical cases with discontinuous barriers have been analysed and the results have been compared with those from both continuous barriers and without barriers cases. The study has been carried out with a dynamic numerical 3D FEM model formulated in the space/time domain, which has previously been validated by authors on the Lisbon-Oporto (Portugal) railway line. The numerical results show the discontinuous barriers with a small separation between axles (less than twice the thickness of the continuous barrier) are an efficient measure in the reduction of vibrations, reaching values of insertion loss of up to $13 \mathrm{~dB}$. Therefore, these could be a very interesting alternative to continuous barriers, in order to conjugate a somewhat lower level of reduction of vibration at a considerable lower cost.
\end{abstract}

Keywords: railway vibrations, mitigation measures, discontinuous barriers, 3D dynamic numerical model, time/space domain.

Introduction

The study of measures to mitigate the vibrations induced by railway traffic is an aspect of railway engineering which dates back to the $19^{\text {th }}$ century. The measures to mitigate vibrations can be classified according to their location (Hung \& Yang, 2001): i) at the source of excitation; ii) in the propagation path; iii) at the receptor. It seems obvious and clear that the actions that improve the geometric characteristics of the vehicles, the wheels and the rail are the most effective as they significantly reduce the dynamic load (Hemsworth, 2000; Nelson, 1996). Nevertheless, the cost of these measures is usually high and it is not always possible to undertake them on service railway lines. However, the measures on the way to the propagation of the waves are usually more cost-effective and can be an interesting option. The effect of these measures is based on the change of the direction of the propagation of the waves on the ground, preventing or reducing the amplitude of the vibrations. In technical literature, various mitigation measures have been studied: techniques for the improvement of the ground (for example, jet grouting) (Coulier at al. 2013a), WIBs (blocks to prevent the propagation of waves), trenches (Barbosa et al., 2014) and piling barriers (Zhang \& Lu, 2013; Lu et al., 2009; Fernández-Ruiz et al., 2020, among others) being the most habitual.

Regarding the measures based on trenches, continuous and discontinuous barriers (or piling), the main conclusion is that their effectiveness is highly dependent on the geometry and the materials used on the barrier and the properties and stratification of the ground (Barbosa et al., 2014). However, studies with contradictory conclusions regarding the best position of these have been found. For example, Çelebi et al. (2009) and Adam \& von Estorff (2005) present that the barriers which are situated far from the rail (passive isolation) are more effective, whilst Çelebi \& Kirtel (2013) present that the barriers which are situated near the rail (active isolation) are more effective. These contradictions may probably be explained by the diversity of the particular characteristics of the ground in each of the cases studied.

Even though there are quite a few studies on continuous barriers or grouting (Barbosa et al., 2014, Coulier et al., 2013a) and their effects seem to be correctly analysed, studies on discontinuous barriers or piling are not abundant. The studies 
carried out by Lu et al. (2009), Zhang \& Lu (2013) and Huang \& Shi (2013), stated that the greater length and stiffness of piling implies amore level of attenuation, as well as being more effective with soft soils. The use of multiple rows of piling also improves the mitigating effect Zhang \& Lu (2013).

The aim of this study is to analyse the level of reduction of vibrations caused by the installation of discontinuous barriers on the ground, by analysing their effect in relation to the classical solution of the continuous barriers and without any barriers. The numerical model used is a 3D FEM model formulated in the space/time domain by means of PLAXIS software. This model has been experimentally validated by the authors Fernández et al. (2017) on the Lisbon-Oporto railway line without the presence of barriers.

\section{Analitical solutions for continuous barriers and discontinuous barriers}

The study of continuous barriers on the ground as a mitigating measure for vibrations has been studied enough (Coulier et al., 2013a, Barbosa et al., 2014). It is well known that Rayleigh waves are responsible for the vibrations induced by railway traffic. So as to study the waves caused by continuous barriers, the decomposition of the Rayleigh waves into plane waves can be previously analysed by following dispersion relation:

$$
\frac{1}{\lambda_{\mathrm{x}}^{2}}+\frac{1}{\lambda_{\mathrm{y}}^{2}}=\frac{1}{\lambda_{\mathrm{R}}^{2}}
$$

where $\lambda_{R}=2 \pi C_{R} / \omega$ is the wavelength of the Rayleigh wave. This decomposition is graphically shown in figure 1a. The propagation of plane waves is characterised by $\lambda_{R} \leq \lambda_{y} \leq \infty$ :

$$
\begin{aligned}
& -\quad \theta=0 \rightarrow \lambda_{x}=\lambda_{R}, \lambda_{y}=\infty \\
& -\quad \theta=\pi / 2 \rightarrow \lambda_{x}=\infty, \lambda_{y}=\lambda_{R}
\end{aligned}
$$

Coulier et al. (2013b) stated that the propagation of these waves on the ground with a wavelength smaller than the wavelength of flexion on the barrier is prevented due to its bending stiffness. This only happens above a critical frequency in which the wavelength of the Rayleigh wave on the ground is equal to the wavelength of the flexural wave on the barrier. The expression of this critical frequency is the following:

$$
\mathrm{f}_{\mathrm{c}}=\frac{\mathrm{C}_{\mathrm{R}}^{2}}{2 \pi} \sqrt{\frac{\rho \mathrm{A}}{\mathrm{EI}}}
$$

where $C_{R}$ is the velocity of the Rayleigh wave, El is the bending stiffness of the barrier, $\rho$ and $A$ are the density and the area of the barrier respectively. The reduction of the level of vibration is only produced for frequencies greater than the critical frequency. This critical frequency decreases as the barrier stiffness increases; therefore, it may be assumed that stiff materials, such as concrete, are appropriate for this type of solutions. In addition to this critical frequency, there is a critical angle where the mitigating effect of the barrier is negligible, which is determined by the ratio between the wavelength of the Rayleigh wave and the wavelength of the flexural wave in the barrier. Its mathematical equation is as follows:

$$
\sin \theta_{c}=\frac{C_{R}}{\sqrt{\omega h}}\left(\frac{12 \rho}{E}\right)^{\frac{1}{4}}
$$

where $\omega$ is the angular frequency and $\mathrm{h}$ is the depth of the barrier. As can be deduced from the previous expression, this critical angle is dependent on the frequency (inversely proportional), on the depth of the barrier (inversely proportional) and the stiffness of the barrier (inversely proportional). This effect is graphically shown in figure $1 \mathrm{~b}$. Thus the greater the depth and stiffness of the barrier, the greater the mitigating effect since the range of frequencies where the reduction is produced is greater and the critical angle is smaller. In the case of discontinuous barriers, there are no analytical solutions available as in the case of continuous barriers due to the complexity of the problem, though there are some mathematical models suggested for this type of barriers, specifically for those formed by piling (Lu et al., 2009; Zhang \& Lu, 2013). These solutions can be considered as semi-analytical solutions. 


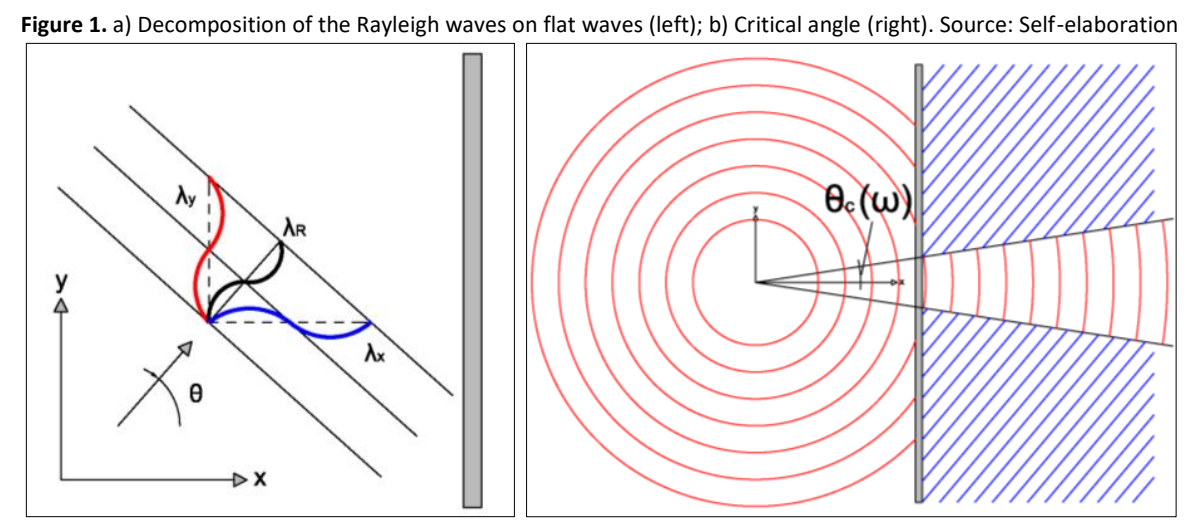

Methodology and Numerical approach

\section{Generalities}

The methodology used in this research is based on the following steps:

- The available information from a real case has been compiled in which railway induced-vibrations have been measured.

- A 3D finite element numerical model has been applied, previously validated by the authors without mitigating measures (Fernández et al. 2017).

- In this 3D numerical model, 3 concrete barriers have been introduced: 1 continuous and 2 discontinuous (with different spacing between axes), obtaining the numerical response for each case.

- The obtained numerical results are compared in the time domain and in the frequency domain through the parameter Insertion Loss (IL), usual in railway-vibration researches about mitigating measures. In this case and for the calculation of the IL, the obtained numerical results without mitigating measures have been taken as the reference (which can be seen in Fernández et al. 2017).

- From the results obtained, the effect of discontinuous concrete barriers for the reduction of railway vibrations is discussed.

The numerical model used in this paper is based on a sub-structured computational scheme: i) the dynamic train-trackground interaction is solved by using a 2D model and the results are used to estimate the transmitted load to each sleeper; ii) the latter is introduced into a numerical 3D Finite Element model formulated in the space/time domain on Plaxis software, where the propagation of waves and ground response is studied. Figure 2 summarises the computational scheme.

\section{Train-track-ground interaction}

The dynamic train-track-ground interaction is calculated according to the model represented in figure 3. It is worth emphasizing that only the vertical movement and the dynamic excitation caused by track irregularities have been considered. For a detailed description of the mathematical formulation, it is recommended to consult Alves Costa et al., 2012a and Alves Costa et al., 2012b.

The equivalent stiffness below sleeper has been calculated by comparison of the static response obtained in both models. The former is the method proposed by Unold and Dischinger (Melis, 2008) and the latter is a 3D numerical model developed in Plaxis. Assuming the same vertical displacement of the rail in the two models, the equivalent stiffness of springs within Unold-Dischinger method is computed, once the numerical result is obtained in Plaxis. The equivalent stiffness below sleeper is then calculated as follows:

$$
\frac{1}{K_{\text {equivalent (ballast,subballast, ground) }}}=\frac{1}{K_{\text {Unold-Dischinger }}}-\frac{1}{K_{\text {railpad }}}
$$


Figure 2. Computational scheme. Source: Self-elaboration.

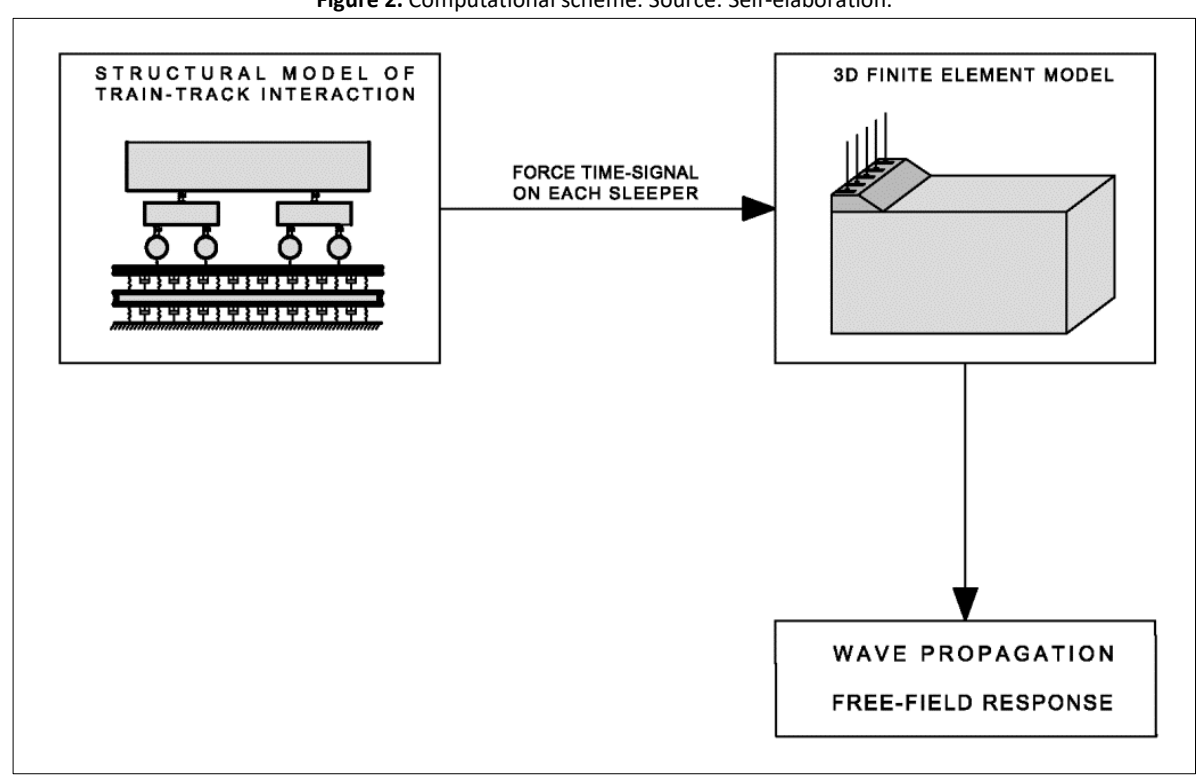

Once the dynamic train-rail-ground interaction is solved, the force on each sleeper $F_{s, i}(t)$ is obtained with the following expression:

$$
F_{s, i}(t)=\left(u_{\text {rail, } i}-u_{\text {sleeper }, i}\right) K_{r p}
$$

where $u_{\text {rail, }}$ and $u_{\text {sleeper, }}$ are the displacements of the track and the sleeper in i position respectively and $K_{r p}$ is the vertical stiffness of the railpad.

Figure 3. Train-track interaction model. Source: Self-elaboration.

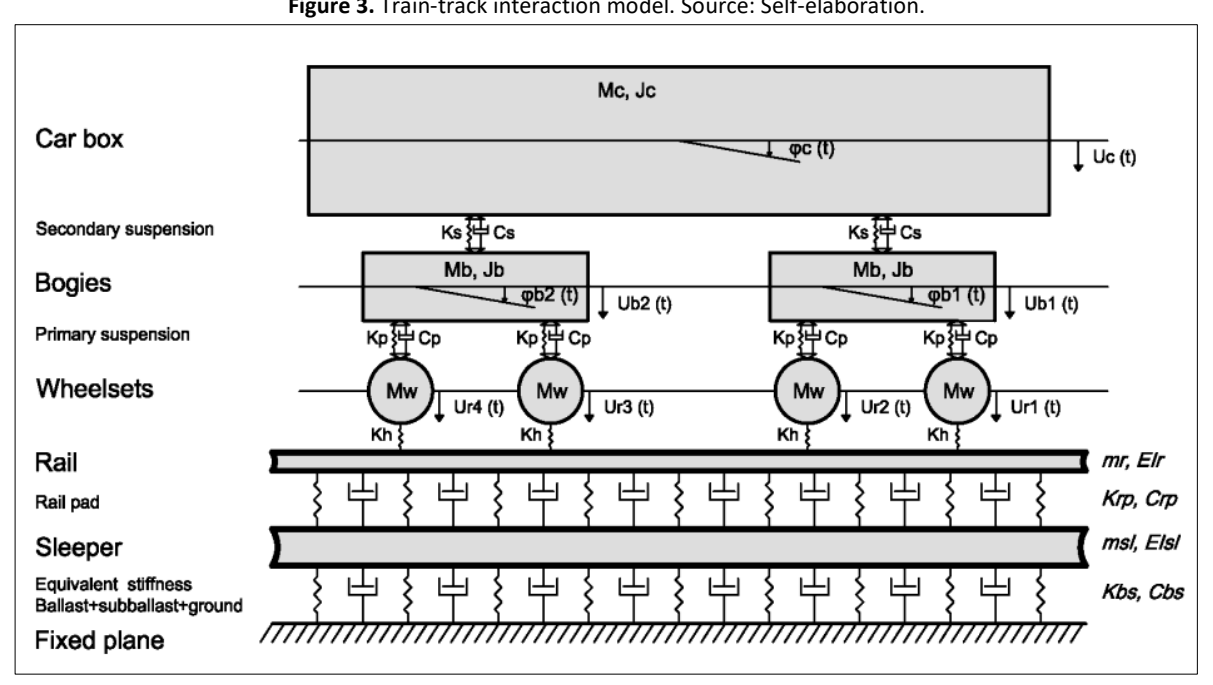

\section{D model of finite elements formulated in the space/time domain}

To simulate the wave propagation in the ground, a 3D FEM model has been used through PLAXIS. This software has been used by authors for the study of railway vibrations, obtaining numerical results in accordance to the ones actually measured. These studies can be consulted in Fernández \& Medina (2015, 2017), Fernández et al. (2019) and in Fernández et al. (2017).

The main characteristics of the model regarding its mathematical formulation are resumed below. Further information can be found in PLAXIS (2011). The basic equation of dynamic equilibrium is written in matrix form as follows:

$$
M \ddot{u}+C \dot{u}+K u=F
$$


where $\mathrm{M}, \mathrm{C}, \mathrm{K}$ and $\mathrm{F}$ are the mass matrix, the damping matrix, the stiffness matrix and load vector respectively. Note that the damping matrix is formulated as a function of the mass and stiffness matrices according to Rayleigh damping, in the following form: $C=\alpha M+\beta K$, where $\alpha$ and $\beta$ are the Rayleigh tuning coefficients.

In PLAXIS code, an implicit time integration scheme of Newmark is applied using the following expressions:

$$
\ddot{u}^{t+\Delta t}=c_{0} \Delta u-c_{2} \dot{u}^{t}-c_{3} \ddot{u}^{t} ; \dot{u}^{t+\Delta t}=c_{1} \Delta u-c_{4} \dot{u}^{t}-c_{5} \ddot{u}^{t} ; u^{t+\Delta t}=u^{t}+\Delta u
$$

where the coefficients $c_{0} \ldots . . . c_{5}$ are expressed as functions of both the time step and the integration parameters $\alpha$ and $\beta$ as follows:

$$
c_{o}=\frac{1}{\alpha \Delta t^{2}} ; c_{1}=\frac{\beta}{\alpha \Delta \mathrm{t}} ; c_{2}=\frac{1}{\alpha \Delta \mathrm{t}} ; c_{3}=\frac{1}{2 \alpha}-1 ; c_{4}=\frac{\beta}{\alpha}-1 ; c_{5}=\frac{\Delta t}{2}\left(\frac{\beta}{\alpha-2}\right)
$$

Different approaches can be used based on the integration parameters although in this paper $\alpha=0.25$ and $\beta=0.5$ have been used because they involve an unconditionally stable analysis (Bathe, 1982). Combining expressions (6), (7) and (8) and taking into account that the expression (6) must be obtained at the end of the time step ( $t+\Delta t$ ), the following general equation for the dynamic analysis can be addressed:

$$
\left(c_{0} M+c_{1} C+K\right) \Delta u=F_{\text {ext }}^{t+\Delta t}+M\left(c_{2} \dot{u}^{t}+c_{3} \ddot{u}^{t}\right)+C\left(c_{4} \dot{u}^{t}+c_{5} \ddot{u}^{t}\right)-F_{\text {int }}^{t}
$$

The boundary conditions correspond to viscous elements based on the proposal by Lysmer \& Kuhlmeyer (1969). The following conditions are imposed on the boundaries:

$$
\begin{aligned}
\sigma_{\mathrm{n}} & =-\mathrm{C}_{1} \rho \mathrm{V}_{\mathrm{p}} \dot{\mathrm{u}}_{\mathrm{n}} \\
\tau & =-\mathrm{C}_{2} \rho \mathrm{V}_{\mathrm{s}} \dot{\mathrm{u}}_{\mathrm{t}}
\end{aligned}
$$

where $\rho$ is the density of the ground and $V_{p}$ and $V_{s}$ are the velocities of the $P$ and $S$ waves, respectively. $C_{1}$ and $C_{2}$ aredimensionless relaxation coefficients. Analytical studies by Lysmer \& Kuhlemeyer (1969) suggest that $C_{1}=C_{2}=1$ implies a perfect absorption when the boundary is achieved by perpendicular impinging waves. As a result, these values are exact only for one-dimensional propagation of body waves. For 2D and 3D cases, perfect absorption depends on angles of incidence. For this reason PLAXIS (2011) recommends to use $C_{1}=1$ y $C_{2}=0.25$ in general cases and these same values have been considered in this research. Moreover, Cohen (1980) has demonstrated that the viscous boundary is not very sensitive to the viscosity coefficients $C_{1}$ and $C_{2}$.

Case study

\section{Introduction}

In order to analyse the mitigating effect of continuous/discontinuous barriers a real case has been chosen, corresponding to the Lisbon-Oporto railway, situated near Carregado (Portugal). Even though there are no barriers in the ground to reduce vibrations, it has been chosen because the authors have validated, with the aid of monitoring data, the numerical model which has been used in this paper for the study of railway vibrations (Fernández et al., 2017). In this way, we have started from a real case in which the mitigating effect of discontinuous and continuous barriers has been numerically studied. A detailed description of the geotechnical characteristics of the site can be consulted in Alves Costa (2011) y Alves Costa et al. (2012b).

\section{Geodynamic properties of the ground}

The characterisation of the ground was carried out by means of surveys, static penetration tests and cross-hole tests. The results of the latter are shown in figure 4. The cross-hole test results have been considered because the seismic wave velocities are obtained with asoil strain magnitude in the same order as those caused by railway traffic, which are usually in the range of very small strains $\left(10^{-5}-10^{-6}\right)$. With regard to the damping of the ground, a type of Rayleigh has been applied which is efficient in models with finite elements formulated in the time domain. As is known, this type of damping depends on the frequency of the waves, consequently, the coefficients $\alpha$ and $\beta$ have been chosen to obtain the most constant damping possible in the range of frequencies of interest, which in this case, has been estimated between 10 and $50 \mathrm{~Hz}$. The resulting damping can be seen in table 1, as well as the elastic properties of the ground. 


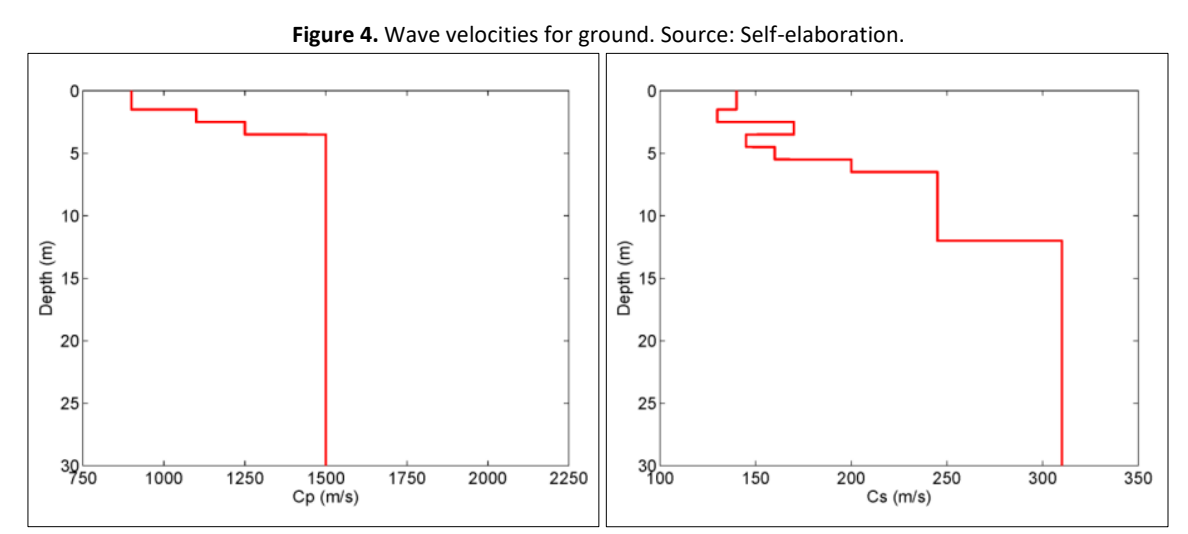

\section{Mechanic properties of the railway track and train characteristics}

Table 2 shows the properties of the elements of the rail which have been considered according to that shown by Alves Costa et al. (2012b). The stiffness of the railpad is $600 \mathrm{kN} / \mathrm{mm}$, the rail is type UIC-60 and the distance between sleepers is 0.6 metres. In addition, table 2 shows the Rayleigh damping for sleeper, ballast and subballast, where the $\alpha$ and $\beta$ coefficients have been selected in order to obtain a "constant damping" in the range of frequencies of interest or main expected frequencies (from $10 \mathrm{~Hz}$ to up $50 \mathrm{~Hz}$ ). Figure 5 shows the profile of track irregularities (left) and the geometry of the Alfa-Pendular train (right), whose properties can be consulted in Fernández et al. (2017). In this case, the train speed has been $164 \mathrm{~km} / \mathrm{h}$.

Table 1. Ground elastic properties. Source: Self-elaboration.

\begin{tabular}{|c|c|c|c|c|c|c|c|c|}
\hline & \multirow{2}{*}{$\begin{array}{c}\text { Thickness } \\
(\mathrm{m})\end{array}$} & \multirow{2}{*}{$\begin{array}{l}\rho \\
\left(\mathrm{kg} / \mathrm{m}^{3}\right)\end{array}$} & \multirow{2}{*}{\multicolumn{2}{|c|}{$E\left(k N / m^{2}\right)$}} & \multirow[t]{2}{*}{$\mathrm{v}$} & \multirow[t]{2}{*}{$\xi(\%)$} & \multicolumn{2}{|c|}{ Rayleigh coefficients } \\
\hline & & & & & & & $\alpha$ & $\beta$ \\
\hline Soil layer 1 & 1.5 & 1900 & \multicolumn{2}{|c|}{$110.8 \times 10^{3}$} & 0.48 & 3 & 4.8 & 0.000152 \\
\hline Soil layer 2 & 1.0 & 1900 & \multicolumn{2}{|c|}{$95.8 \times 10^{3}$} & 0.49 & 3 & 4.8 & 0.000152 \\
\hline Soil layer 3 & 1.0 & 1900 & \multicolumn{2}{|c|}{$163.7 \times 10^{3}$} & 0.49 & 3 & 4.8 & 0.000152 \\
\hline Soil layer 4 & 1.0 & 1900 & \multicolumn{2}{|c|}{$119.5 \times 10^{3}$} & 0.49 & 3 & 4.8 & 0.000152 \\
\hline Soil layer 5 & 1.0 & 1900 & \multicolumn{2}{|c|}{$145.4 \times 10^{3}$} & 0.49 & 3 & 4.8 & 0.000152 \\
\hline Soil layer 6 & 1.0 & 1900 & \multicolumn{2}{|c|}{$226.6 \times 10^{3}$} & 0.49 & 3 & 4.8 & 0.000152 \\
\hline Soil layer 7 & 5.5 & 1900 & \multicolumn{2}{|c|}{$339.0 \times 10^{3}$} & 0.48 & 3 & 4.8 & 0.000152 \\
\hline Soil layer 8 & 18.0 & 1900 & \multicolumn{2}{|c|}{$539.6 \times 10^{3}$} & 0.47 & 3 & 4.8 & 0.000152 \\
\hline & \multirow{2}{*}{\multicolumn{2}{|c|}{$\rho\left(\mathrm{kg} / \mathrm{m}^{3}\right)$}} & \multirow[t]{2}{*}{$E\left(k N / m^{2}\right)$} & $v$ & \multirow{2}{*}{\multicolumn{2}{|c|}{$\xi(\%)$}} & \multicolumn{2}{|c|}{ Rayleigh coefficients } \\
\hline & & & & & & & $\alpha$ & $\beta$ \\
\hline Sleeper & \multicolumn{2}{|c|}{2500} & $30 \times 10^{6}$ & 0.20 & \multicolumn{2}{|c|}{1} & 1.67 & 0.000053 \\
\hline Ballast & & & $97 \times 10^{3}$ & 0.12 & & & 8.94 & 0.000283 \\
\hline Subballast & & & $212 \times 10^{3}$ & 0.20 & & & 6.14 & 0.000195 \\
\hline
\end{tabular}

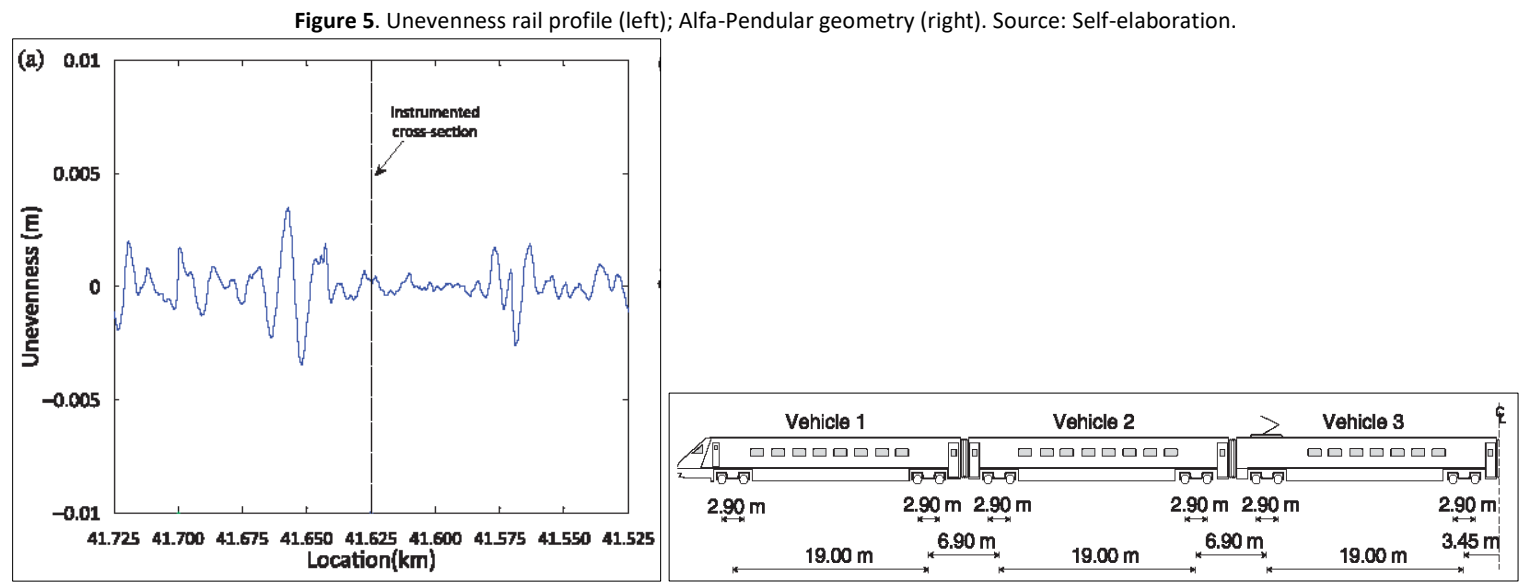




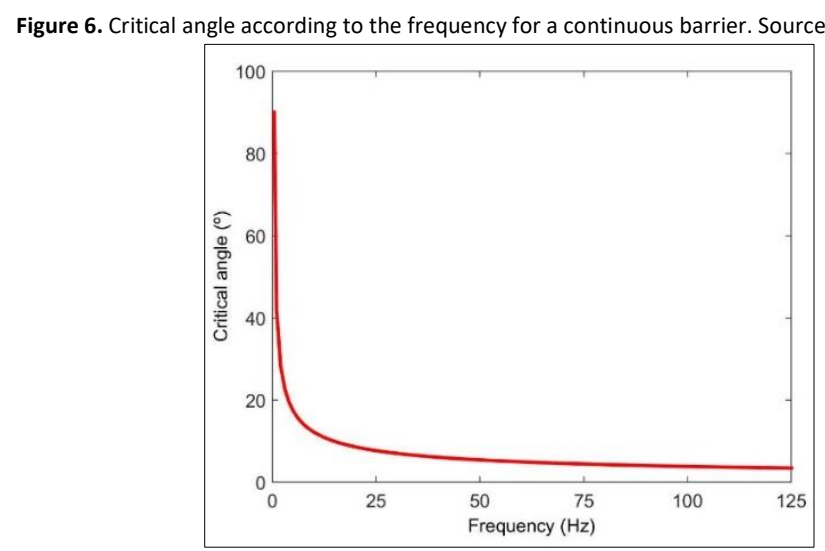

\section{Studied barriers}

All the studied cases correspond to measurements of passive isolation, since they are located far from the track (20 metres). Two discontinuous barriers have been analysed: one barrier with 1 metre thickness and a 2 metre distance between axles and 7 metre depth (named discontinuous barrier 1); one barrier with the same thickness and depth as the previous one, but a 3 metre distance between axles (named discontinuous barrier 2 ). In order to be able to compare the results obtained in these cases with continuous barriers the following case has also been modelled: one continuous barrier with 1 metre width and 7 metre depth. In all cases the barrier material is concrete and its properties are the same as the ones considered for the sleepers (see table 2). For this case, the analytic solution for continuous barrier has a critical frequency of $0.45 \mathrm{~Hz}$ and a critical angle $\left(\theta_{c}\right)$ which is shown in figure 6 . A schematic plan view of the 3 studied barriers is shown in figure 7 .

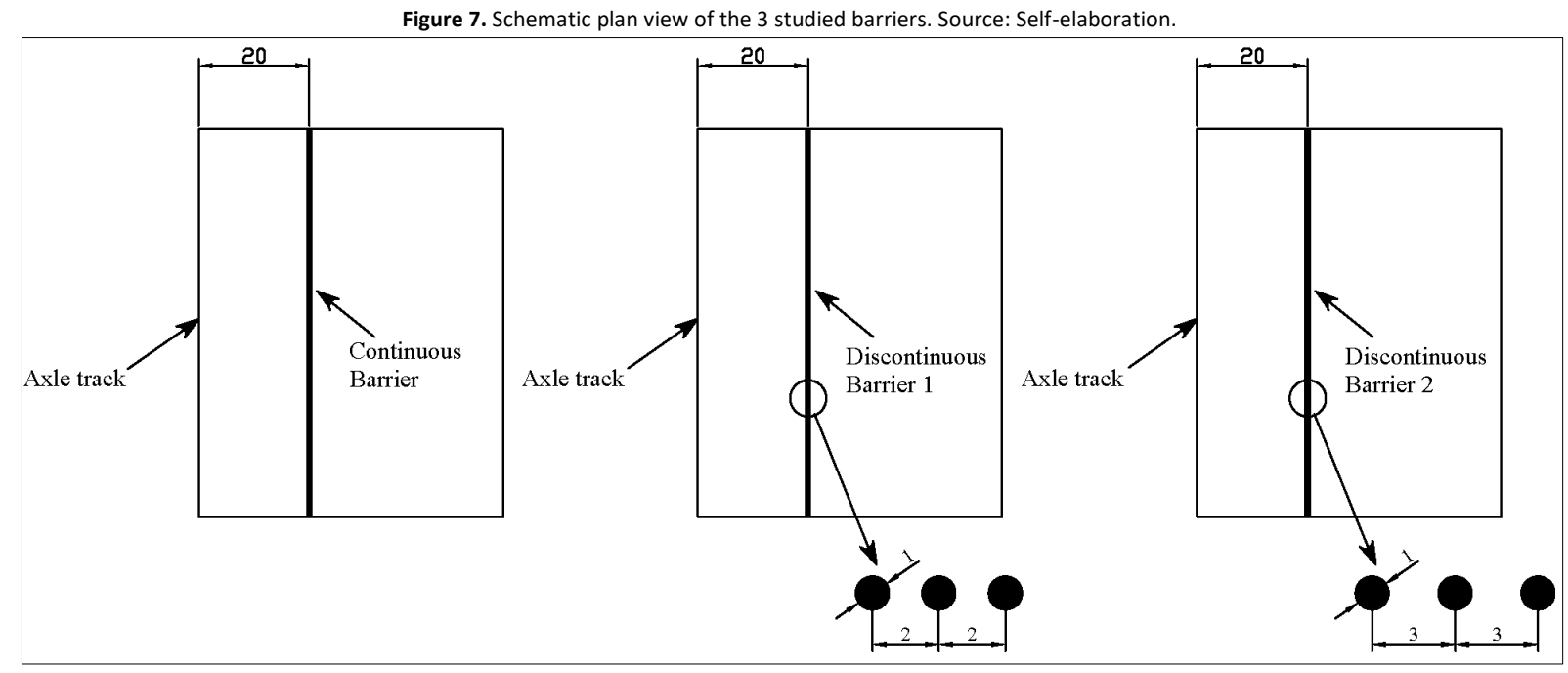

\section{Description of the numerical model}

For the numerical modelling of moving loads, a 30-metre-long finite element mesh has been considered, in which the loads have been applied on the sleepers. This consideration has been experimentally validated by authors and can be consulted in Fernández et al. (2017). In addition, it has also been numerically validated comparing it to 2.5D models formulated in the wavenumber/frequency domain, which can be also consulted in Fernández et al. (2017). The length of numerical model is $70 \mathrm{~m}$ whereas the total length of the train is higher than $100 \mathrm{~m}$. This fact is common in numerical models to simulate railway vibrations and it does not cause a loss of accuracy (Thompson et al. 2016; Hall, 2003; Abu Sayeed \& Shahin, 2016, among others). The dynamic time step is $6.25 \times 10^{-5} \mathrm{~s}$, which is determined by the size of the finite elements of the sleeper. Figures 8 and 9 show the numerical model used in this paper. Furthermore, figure 10 shows the cases of discontinuous and continuous barriers considered. Four control points have been selected for the comparison of numerical results, all of them situated in the centre of the numerical model and at 22.5, 30, 37.5 and 45 metres distance from the axle of the track. 
Figure 8. 3D numerical model: three-dimensional view (left); plan (right) (in metres). Source: Self-elaboration.
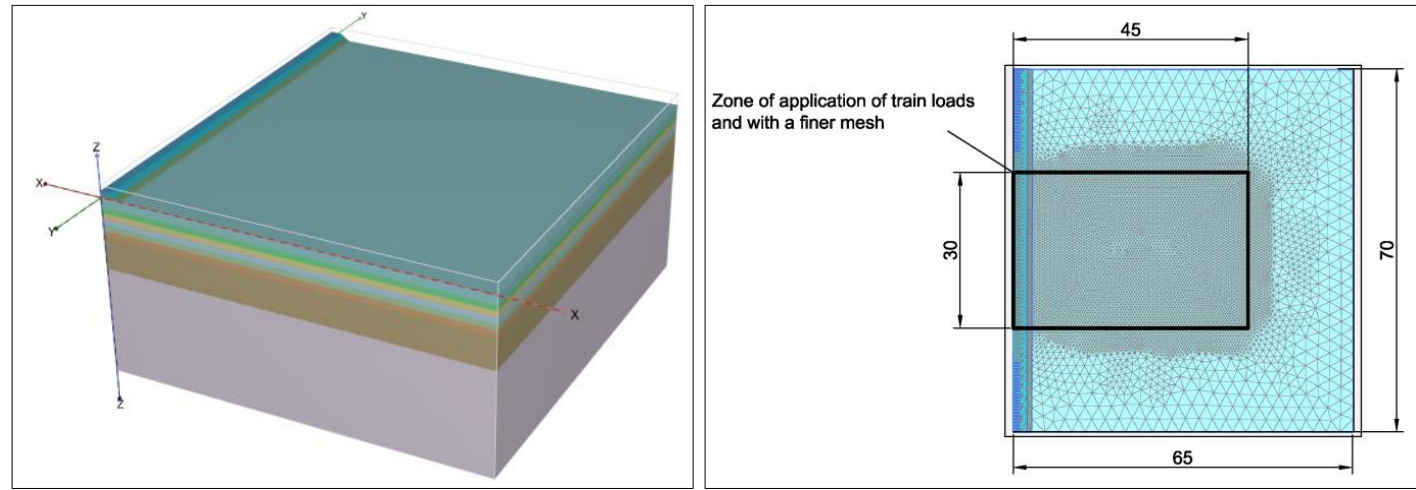

Figure 9. Finite element mesh: general section (left); track (right).Dimensions are in meters. Source: Self-elaboration.

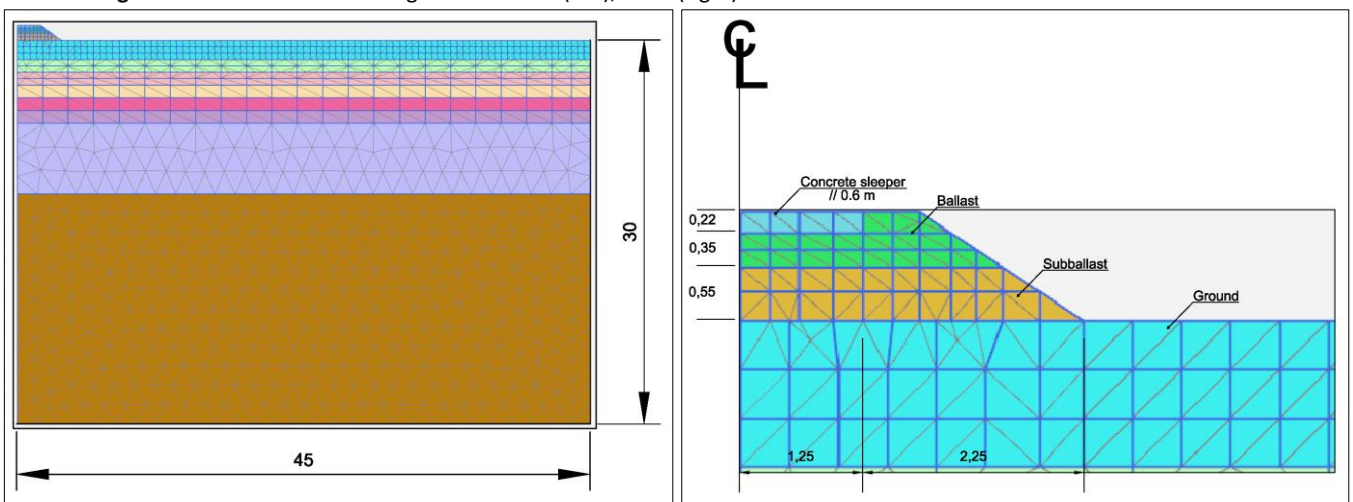

Figure 10. 3D numerical model: with continuous barrier (top left); with discontinuous barrier 1 (top right); with discontinuous barrier 2 (down). Source: Self-elaboration.
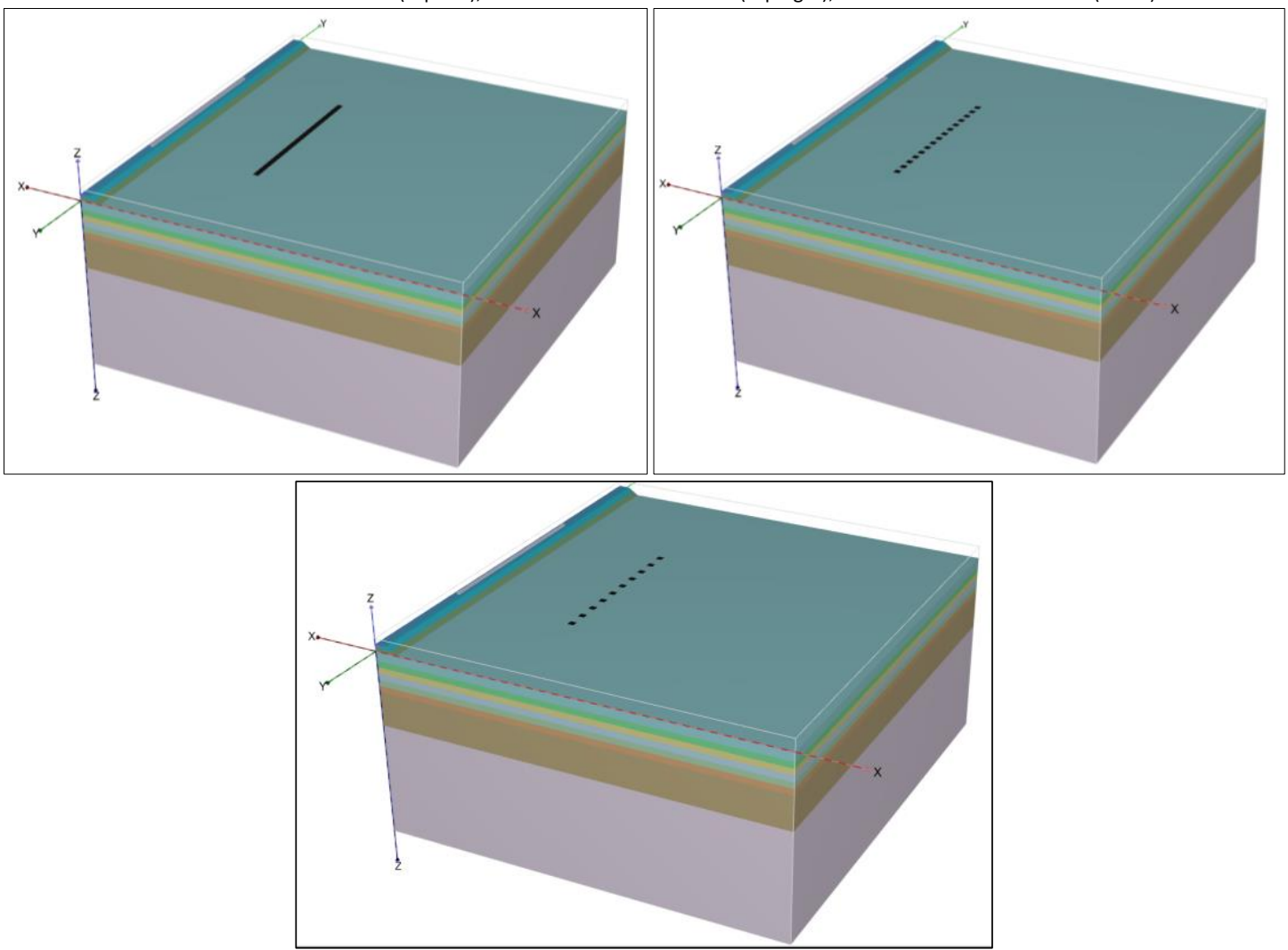
Figure 11 shows the time record of the vertical velocity. It can be seen as the continuous barrier is the most effective solution. In fact, it shows the lowest vibration levels of the 3 proposed solutions in all of the considered control points. As expected, an increase in the separation between axles in the discontinuous barrier has a negative mitigating effect, being the solution with the largest distance between axes the less effective, at least in an analysis in the time domain. There are several relevant facts that deserve to be analyzed carefully. One of them is that the differences between the 3 solutions are clearly appreciable in the control points closest to the barrier $(22.5$ and 30 meters from the axis of the track) with differences in the peak velocity values of almost $100 \%$. However, these differences are far less important in the furthest points from the barrier, being practically negligible at the point located 45 meters from the axis of the track.

Figure 11. Time record of vertical velocity at different distances: a) $22.5 \mathrm{~m}$; (b) $30 \mathrm{~m}$; (c) $37.5 \mathrm{~m}$; (c) $45 \mathrm{~m}$ (red line: continuous barrier; blue line: discontinuous barrier 1 ; green line: discontinuous barrier 2). Source: Self-elaboration.

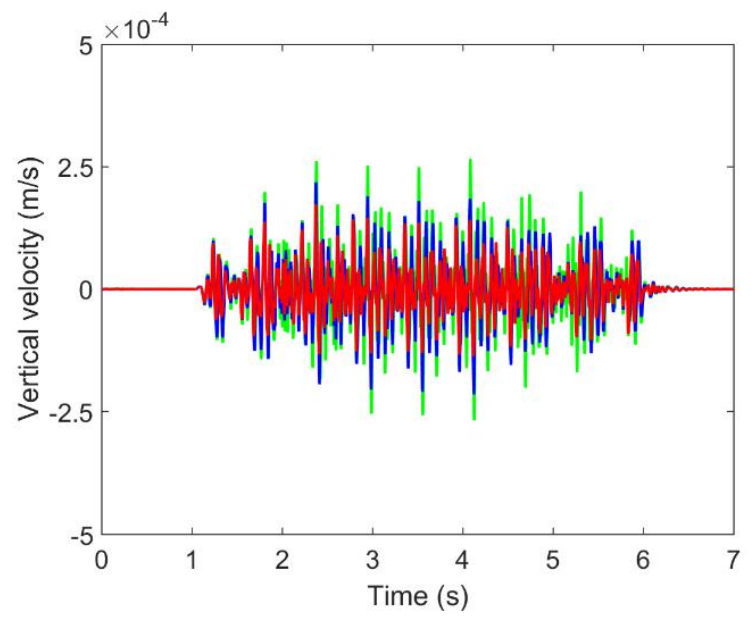

C

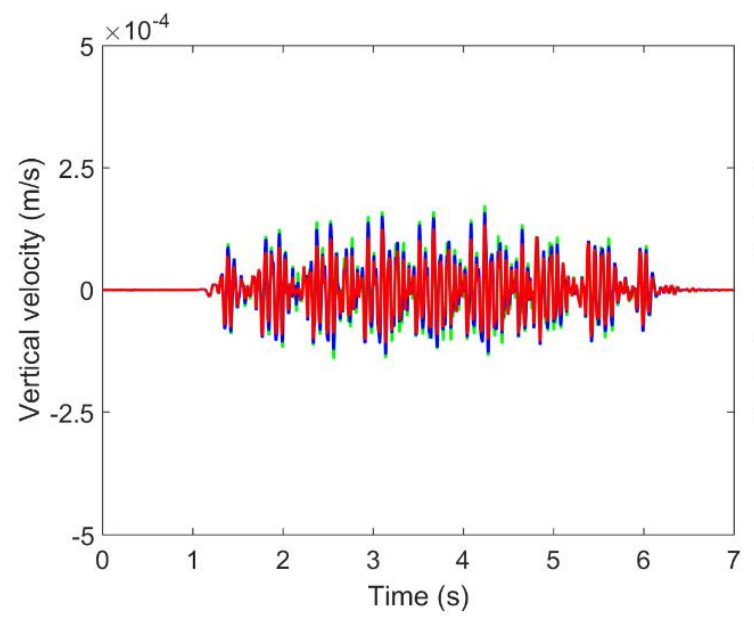

b

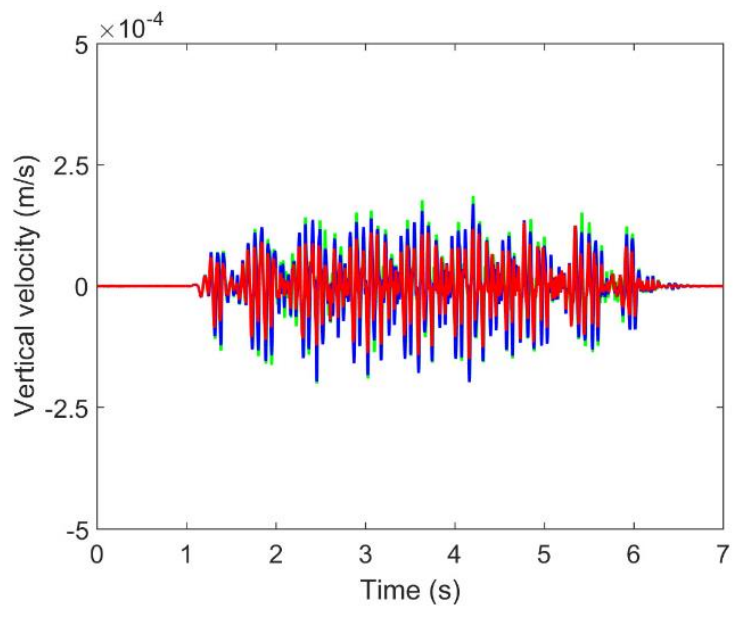

d

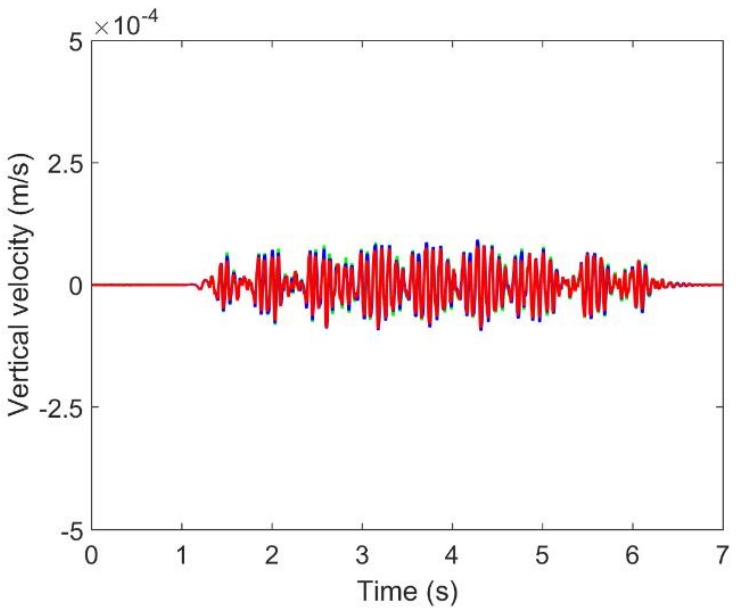

So as to be able to appropriately compare the numerical results obtained, they are also shown in third octave and in terms of insertion loss $(\mathrm{dB})$ in figure 12 . This is defined as follows:

$$
\mathrm{IL}(\mathrm{dB})=20 \log _{10} \frac{\left|\mathrm{v}_{\text {ref }}\right|}{|\mathrm{v}|}
$$

where $v_{\text {ref }}$ corresponds to the numerical case without barrier. It has to be emphasized that this last case coincides with the numerical model proposed by the authors (Fernández et al. 2017).

As can be observed, the discontinuous barriers provide a reduction in the level of vibration all over the frequency ranges and points analysed. This is relevant in that, to a greater or lesser extent, these solutions can be described as effective in mitigating the vibrations. In addition, two important issues can be highlighted: the first one corresponds to the fact that the tendency in insertion loss of discontinuous barriers is very similar; and the second one is that at greater distances from the axis of the track the reduction is less. It is also significant that at all the analysed points the insertion 
loss is greater in the range of frequencies between 10 and $70 \mathrm{~Hz}$, due basically to the fact that outside this range, the energy of vibration is smaller, as can be seen in detail in figure $12 a, 12 b, 12 c$ y $12 d$.

Figure 12. Insertion loss (IL) at distinct distances: a) $22.5 \mathrm{~m}$; (b) $30 \mathrm{~m}$; (c) $37.5 \mathrm{~m}$; (c) $45 \mathrm{~m}$ (red line: continuous barrier; blue line: discontinuous barrier1; green line: discontinuous barrier 2). Source: Self-elaboration
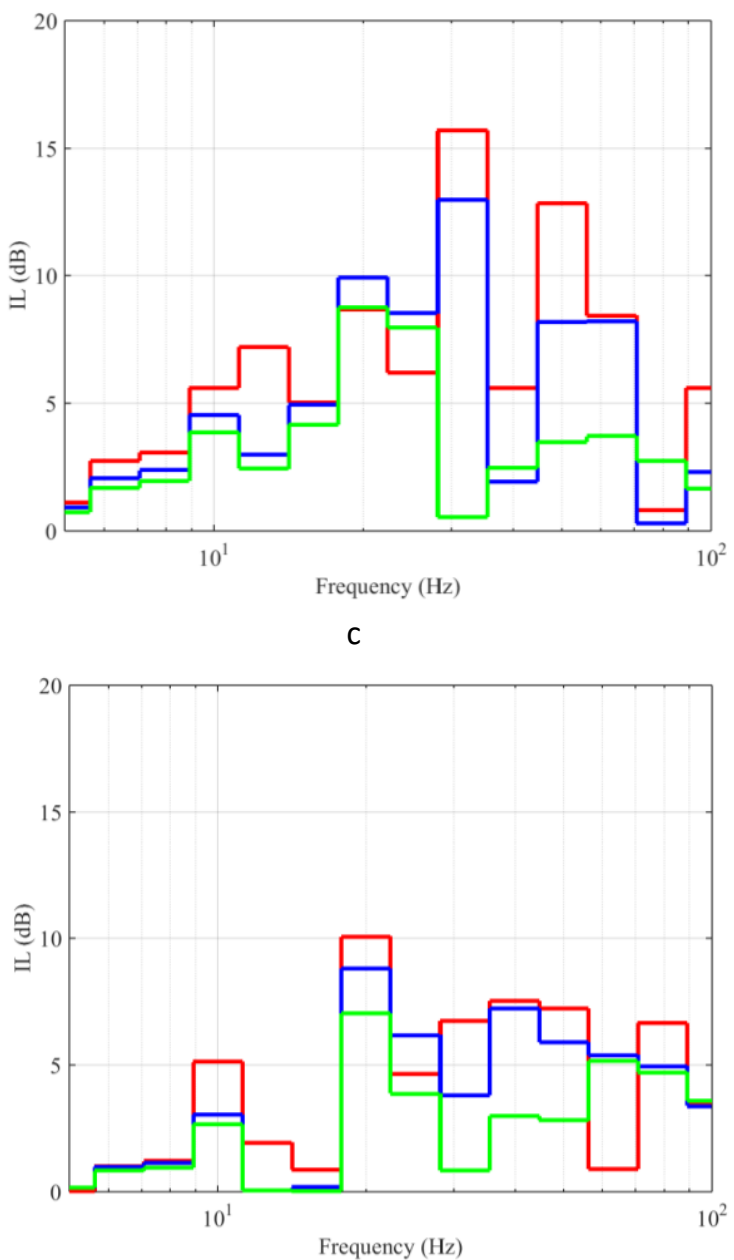

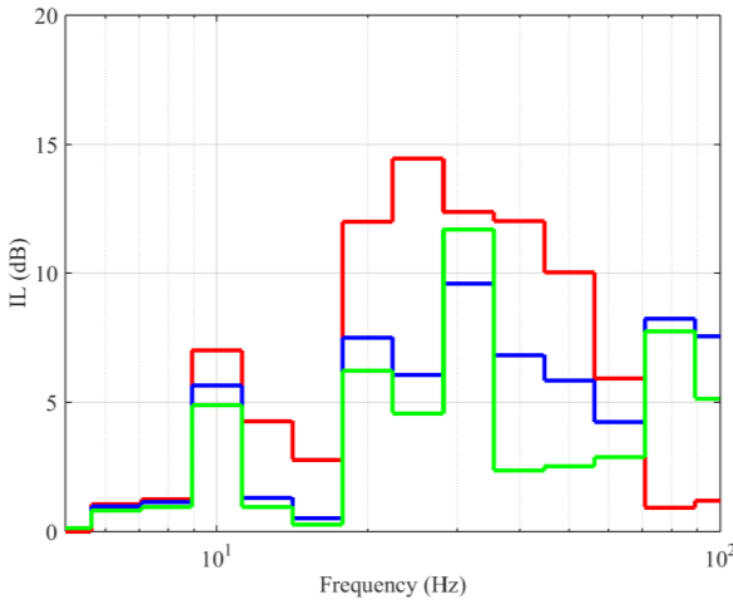

d

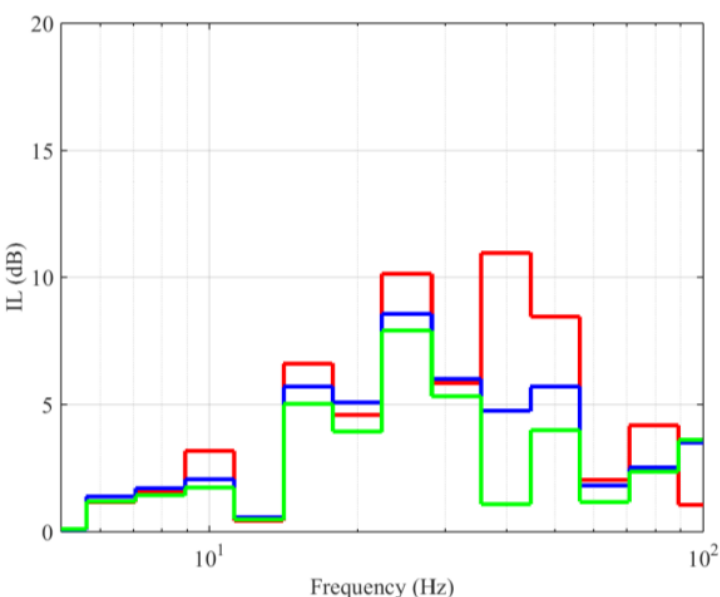

In quantitative terms, the discontinuous barrier 1 causes a maximum reduction of $13 \mathrm{~dB}$ for the point situated at 22.5 metres and in the $31.5 \mathrm{~Hz}$ frequency band. Moreover, in the range of frequencies between 16-63 Hz, the reduction is always greater than $5 \mathrm{~dB}$ at all considered points, which is a quite acceptable level of reduction. In the case of the discontinuous barrier 2 , the maximum reduction is $8 \mathrm{~dB}$ for the point situated at 22.5 metres and in the 20-25 $\mathrm{Hz}$ frequency band. In the case of discontinuous barrier 2, the reduction of vibrations is smaller than in the discontinuous barrier 1, with insertion loss values between 2 to $4 \mathrm{~dB}$ in the 16-63 $\mathrm{Hz}$ frequency ranges.

When comparing the solutions of discontinuous barriers with the continuous barrier case, it is observed that, in general, it also shows a similar tendency in terms of insertion loss and it is somewhat more effective in terms of reduction of vibrations. In fact, the differences between continuous and discontinuous barrier 1 vary from 1 to $4 \mathrm{~dB}$ in the range of frequencies $0-80 \mathrm{~Hz}$, whilst for the discontinuous barrier 2 , the differences with the continuous barrier are greater, from 1 to $9 \mathrm{~dB}$. These differences are more relevant in the case of the discontinuous barrier 2 , where a very low level of reduction starts to be appreciated, whereas that in the case of the discontinuous barrier 1 the differences with the continuous barrier are not very important. Though the most efficient solution is the continuous barrier, discontinuous barriers also present interesting values of insertion loss, being decisive the separation between axles, in order to achieve a greater level of reduction.

It is also worth noting that in the low frequency range (up to 20-25 Hz) the differences between continuous and discontinuous barriers are low in all the analysed points, being the greatest differences in the frequency range 25-70 $\mathrm{Hz}$. This fact could be explained from the relationship between frequency and wavelength. In this way, the low frequency waves (large wavelengths) pass through the barriers without being affected while the medium and high frequency waves (small wavelengths) are affected by the barriers, especially those in which the axle separation is smaller. 
As previously mentioned, it is an evident fact that the tendency in the curves of insertion loss is relatively similar in the 3 cases considered, which could explain the phenomenon that is produced with discontinuous barriers, where the lack of longitudinal continuity can be one of the causes for the lower reduction of vibrations. In fact, the reducing effect which occurs in the continuous barriers is mainly due to the fact that the incident waves are partially "trapped" by the barrier and directed longitudinally throughout the barrier, involving a significant reduction of vibrations. Nevertheless, continuous barriers are technical solutions with a higher cost than discontinuous barriers, which means that, according to the required level of reduction of vibrations, these can be an appropriate alternative when the distance between axles is not very large. In this research, increasing the distance from 2 to 3 metres has lowered the efficiency between $20 \%$ and $50 \%$ in terms of insertion loss.

\section{Conclusions}

In this paper, discontinuous concrete barriers for the reduction of vibrations caused by railway traffic as passive isolation measures have been studied in comparison with continuous barriers. For this purpose, two cases of discontinuous barriers with separations between axles of 2 and 3 metres have been studied and have been compared with the case of a continuous barrier. A numerical approach, which was experimentally validated, based on 3D FEM model formulated in the space/time domain, has been used for this study.

The numerical results show that discontinuous barriers are efficient measures for the mitigation of railway vibrations, reaching values of insertion loss of up to $13 \mathrm{~dB}$. However, very close attention should be paid to the separation between axles since it plays a very relevant role in the level of reduction of vibrations. In the cases analysed in this paper, when varying the separation between the axles from 2 to 3 metres a loss of efficiency between $20 \%$ to $50 \%$ has been obtained, in terms of insertion loss.

The obtained results have been compared to the case of the continuous barrier, showing differences with little relevance (between 1 and $4 \mathrm{~dB}$ ) in the case of the discontinuous barrier with a 2 metre separation between axles. Nevertheless, more relevant differences have been obtained in the case of a 3 metre separation between axles ( 1 to $9 \mathrm{~dB}$ ). This leads to conclude that discontinuous barriers with a small separation between axles (less than twice the thickness of the barrier) are an efficient measure in the reduction of vibrations and a very interesting alternative to continuous barriers, in order to conjugate a somewhat lower level of reduction of vibration at a considerable lower cost.

\section{References}

Abu Sayeed, Md. \& Shahin, M.A. (2016). Three-dimensional numerical modelling of ballasted railway track foundations for high-speed trains with special reference to critical speed. Transportation Geotechnics, 6,55-65.

Adam M., \& von Estorff, O. (2005). Reduction of train-induced building vibrations by using open and filled trenches. Computers \& Structures, 83 (1), 11-24.

Alves Costa, P. (2011). Vibrations of track-ground system induced by railway traffic. Numerical modeling and experimental validation. Faculty of Engineering, Porto: University of Porto, (in Portuguese).

Alves Costa, P., Calçada, R., \& Silva Cardoso, A. (2012a). Influence of train dynamic modeling strategy on the prediction of track-ground vibrations induced by railway traffic. Proc Inst Mech Eng, Part F: Journal of Rail and Rapid Transit, 226 (4), 434-450.

Alves Costa, P., Calçada, R., \& Silva Cardoso, A. (2012b). Track-ground vibrations induced by railway traffic: in-situ measurements and validation of a 2.5D FEM-BEM model. Soil Dynamics and Earthquake Engineering, 32, 111-128.

Barbosa, J., Alves Costa, P., \& Calçada, R. (2014). Abatement of railway vibrations: Numerical comparison of trench solutions. Engineering Analysis with Boundary Elements, 55, 122-139.

Bathe, K. J. (1982). Finite Element Procedures in Engineering Analysis. Prentice Hall, Inc., Englewood Cliffs, New Jersey.

Çelebi, E., Firat, S., Beyhan, G., Çankaya, I., Vural, I., \& Kirtel, O. (2009). Field experiments on wave propagation and vibration isolation by using wave barriers. Soil Dynamics and Earthquake Engineering, 29(5), 824-833.

Çelebi, E., \& Kirtel, O. (2013). Non-linear 2-D FE modeling for prediction of screening performance of thin-walled trench barriers in mitigation of traininduced ground vibrations. Construction and Building Materials 42, 122-131.

Cohen, M. (1980). Silent boundary methods for transient wave analysis. California Institute of Technology. Pasadena (California).

Coulier, P., François, S., Degrande, G., \& Lombaert, G. (2013a). Subgrade stiffening next to the track as a wave impeding barrier for railway induced vibrations. Soil Dynamics and Earthquake Engineerin, 48, 119-131. 
Coulier, P., François, S., Degrande, G., \& Lombaert, G. (2013b). Mitigation of railway induced vibrations using stiff wave barriers. 6th International Symposium on Environmental Vibration (ISEV2013), Shanghai (China).

Fernández Ruiz, J. \& Medina, L. (2015). Application of an advanced soil constitutive model to the study of railway vibrations in tunnels through 2D numerical models: a real case in Madrid (Spain). Revista de la Construcción, 14 (3), 55-63.

Fernández Ruiz, J., Alves Costa, P., Calçada, R., Medina Rodríguez, L., \& Colaço, A. (2017). Study of ground vibrations induced by railway traffic in a 3D FEM model formulated in the time domain: experimental validation. Structure and Infrastructure Engineering, 13 (5), $652-664$

Fernández Ruiz, J., \& Medina, L. (2017). Methodology for predicting vibrations induced by underground railways in 2D numerical models: a real case in Madrid, Spain. Revista Ingeniería de Construcción, 32 (3), 173-184.

Fernández Ruiz, J., Soares, P. J., Alves Costa, P., \& Connolly, D. P. (2019). The effect of tunnel construction on future underground railway vibrations. Soil Dynamics and Earthquake Engineering, 125,105756

Fernández-Ruiz, J., Medina Rodríguez, L.E., Costa, P.A. (2020). Use of Tyre-Derived Aggregate as Backfill Material for Wave Barriers to Mitigate RailwayInduced Ground Vibrations. International Journal of Environmental Research and Public Health, 17, 9191.

Hall L (2003). Simulations and analyses of train-induced ground vibrations in finite element models. Soil Dynamics and Earthquake Engineering, 23, 403-413.

Hemsworth, B. (2000). Reducing groundborne vibrations: state-of-the-art study. Journal of Sound and Vibration 231(3), 703-709.

Huang, J., \& Shi, Z. (2013). Attenuation zones of periodic pile barriers and its application in vibration reduction for plane waves. Journal of Sound and Vibration, $332(19), 4423-4439$.

Hung, HH., \& Yang, Y.B. (2001). A review of researches on ground-borne vibrations with emphasis on those induced by trains. Proceedings of the National Science Council. Part A: Physical Science and Engineering, 25(1), 1-16.

Lu, J., Xu. B., \& Wang, J. (2009). A numerical model for the isolation of moving-load vibrations by pile rows embedded in layered porous media. International Journal of Solids and Structures, 46, 3771-3781.

Lysmer, J., \& Kuhlmeyer, R.L. (1969). Finite dynamic model for infinite media. Journal of the Engineering Mechanics, 95, 859-877.

Melis, M. (2008). Apuntes de introducción a la dinámica vertical de la vía y a las señales digitales en ferrocarriles. 1a Edición; Madrid (España); Cátedra de ferrocarriles, E.T.S. de Ingenieros de Caminos, Canales y Puertos, Universidad Politécnica de Madrid; ISBN: 978-84-612-7686-8. (In Spanish)

Nelson, J. (1996). Recent developments in ground-borne noise and vibration control. Journal of Sound and Vibration, 193(1), 367-376.

PLAXIS. (2011). Reference and scientific manual 3D. Delft,http://www.plaxis.nl/plaxis3d/manuals/

Thompson, D.J., Jiang, J., Toward, M.G.R., Hussein, M.F.M., Ntotsios, E., Kijckmans, A., Coulier, P., Lombaert, G., \& Degrande, G. (2016). Reducing railway-induced ground-borne vibration by using open trenches and soft-filled barriers. Soil Dynamics and Earthquake Engineering, $88,45-59$.

Zhang, X., \& Lu., J. (2013). A wavenumber boundary element method model for the simulation of vibration isolation by periodic pile rows. Engineering Analysis with Boundary Elements, 37(7-8), 1059-1073. 